\title{
SHORT COMMUNICATION Diode Characteristics of a Metal-Insulator-Metal Structure
}

\author{
S. K. GUPTA, C. M. SINGAL and V. K. SRIVASTAVA \\ Department of Physics, University of Roorkee, Roorkee, India
}

\begin{abstract}
The large intrinsic field that can exist inside a thin insulator film which is sandwiched between dissimilar electrodes, is utilized to make the MIM (Metal-Insulator-Metal) structure operate as a diode. Dielectric monomolecular films of barium stearate are sandwiched between an aluminium and a tin electrode. The maximum value of forward bias current is of the order of a few nanoamperes. It is suggested that the value of current may be increased by utilizing a suitably doped dielectric.
\end{abstract}

\section{INTRODUCTION}

An important class of thin film diodes are the Schottky diodes in which a semiconductor is contacted with a metal or any other material which may form a potential barrier at the contact. In the present work we have utilized the fact that there exists an intrinsic field inside a thin dielectric film sandwiched between two metals having different work functions ${ }^{1}$. When a bias is applied between these electrodes, it enhances the current in one direction and blocks it in the other depending upon the direction of the intrinsic field due to the two metal electrodes.

\section{EXPERIMENTAL}

The test samples of aluminium-barium stearate-tin structure were prepared using the following procedure:

Aluminium strips of $1 \mathrm{~mm}$ width were deposited onto well-cleaned glass slides in a high vacuum coating unit (vacuum $\sim 10^{-6} \mathrm{~mm} \mathrm{Hg}$ ). In order to deposit a barium-stearate film by the BlodgettLangmuir technique ${ }^{2,3,4}$, a barium-chloride solution (conc. $3 \times 10^{-5} \mathrm{M}$ ) was made in doubly distilled water in a Langmuir trough. The $\mathrm{pH}$ was adjusted to about 7.2 by adding $4 \times 10^{-4} \mathrm{M}$ of potassium bicarbonate. A monomolecular layer of stearic acid was spread on the surface of the solution whose boundary was defined by a waxed thread. The barium stearate film was kept under constant compression by a 'piston oil' monolayer. When the slide is dipped and withdrawn from the solution vertically, two monolayers are deposited on the slide with the barium stearate molecules oriented perpendicular to the surface of the slide. The process can be repeated for increasing the number of monolayers in the deposited film. The upper tin electrode was again deposited by the evaporation technique (vacuum $\sim 10^{-6} \mathrm{~mm} \mathrm{Hg}$ ). The capacitor area was approximately $1 \mathrm{~mm}^{2}$.

The measurements of current on the above prepared samples, as a function of applied bias, were made by an electrometer amplifier. Since very low currents are involved the whole assembly, including the low voltage supply, was electrically shielded to avoid any stray signal effects.

\section{RESULTS AND DISCUSSION}

In Figure 1 current versus voltage for a four layer barium stearate sandwich is plotted. The sandwich behaves like a diode in the voltage range $-0.6 \mathrm{~V}$ to $+0.6 \mathrm{~V}$. The maximum backward bias current is $0.2 \mathrm{nA}$. The characteristics of the diode structure are explained as follows:

The dielectric is sandwiched between dissimilar electrodes, so there exists an intrinsic voltage due to the work function difference of two metals. The work function of tin is higher than that of aluminium $^{1}$, giving an intrinsic field in the insulating film directed from aluminium to tin. Thus when the tin electrode is negatively biased the current increases and then becomes unstable. If the tin electrode is positively biased the applied voltage neutralizes the internal voltage and the current decreases and becomes zero for a voltage which is nearly equal to the internal voltage. If the tin electrode is made more positive, the current starts 


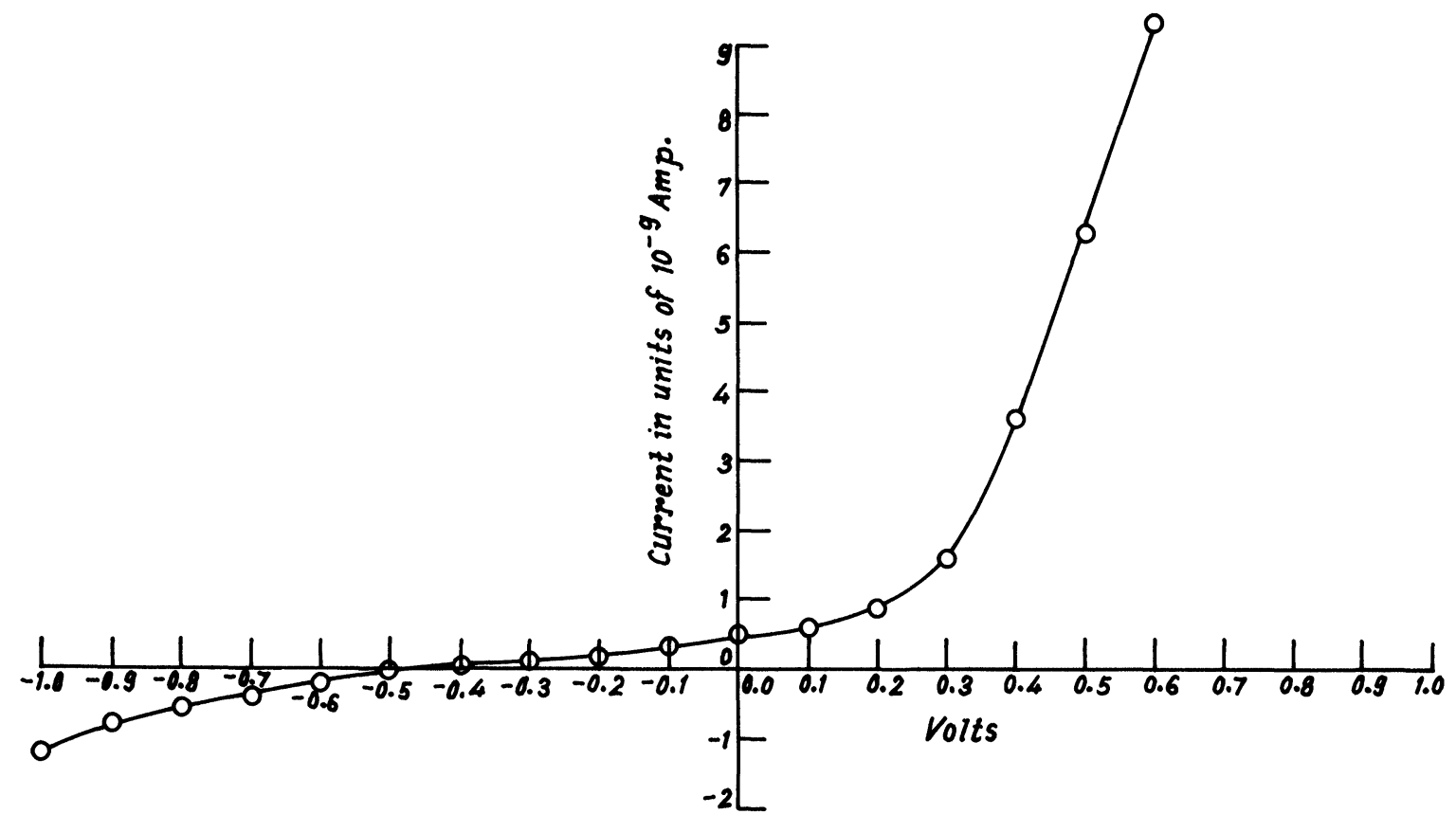

FIGURE 1 Diode characteristics of an aluminium-four layer barium stearate-tin structure.

flowing in the backward direction. Thus the operating voltage range $( \pm 0.6 \mathrm{~V})$ of the diode is nearly equal to the internal voltage of the structure which for this particular diode was found to be $0.6 \mathrm{~V}$.

In the present diode system the value of the current is very low but this can be increased if a highly doped dielectric is used. The working voltage range of this diode may be increased by choosing suitable metals for the upper and lower electrodes which have larger work function difference.

\section{ACKNOWLEDGEMENTS}

The authors (S. K. Gupta and C. M. Singal) wish to acknowledge the financial support provided by Council of Scientific and Industrial Research, India.

\section{REFERENCES}

1. J. G. Simmons, Phys. Rev. Letts. 10, 10 (1963).

2. K. B. Blodgett, J. Am. Chem. Soc. 57, 1007 (1935).

3. K. B. Blodgett and I. Langmuir, Phys. Rev. 51, 964 (1937).

4. V. K. Srivastava Physics of Thin Films. Vol. 7, p. 311, Eds. G. Hass and R. Thun, (Academic Press, New York 1973). 

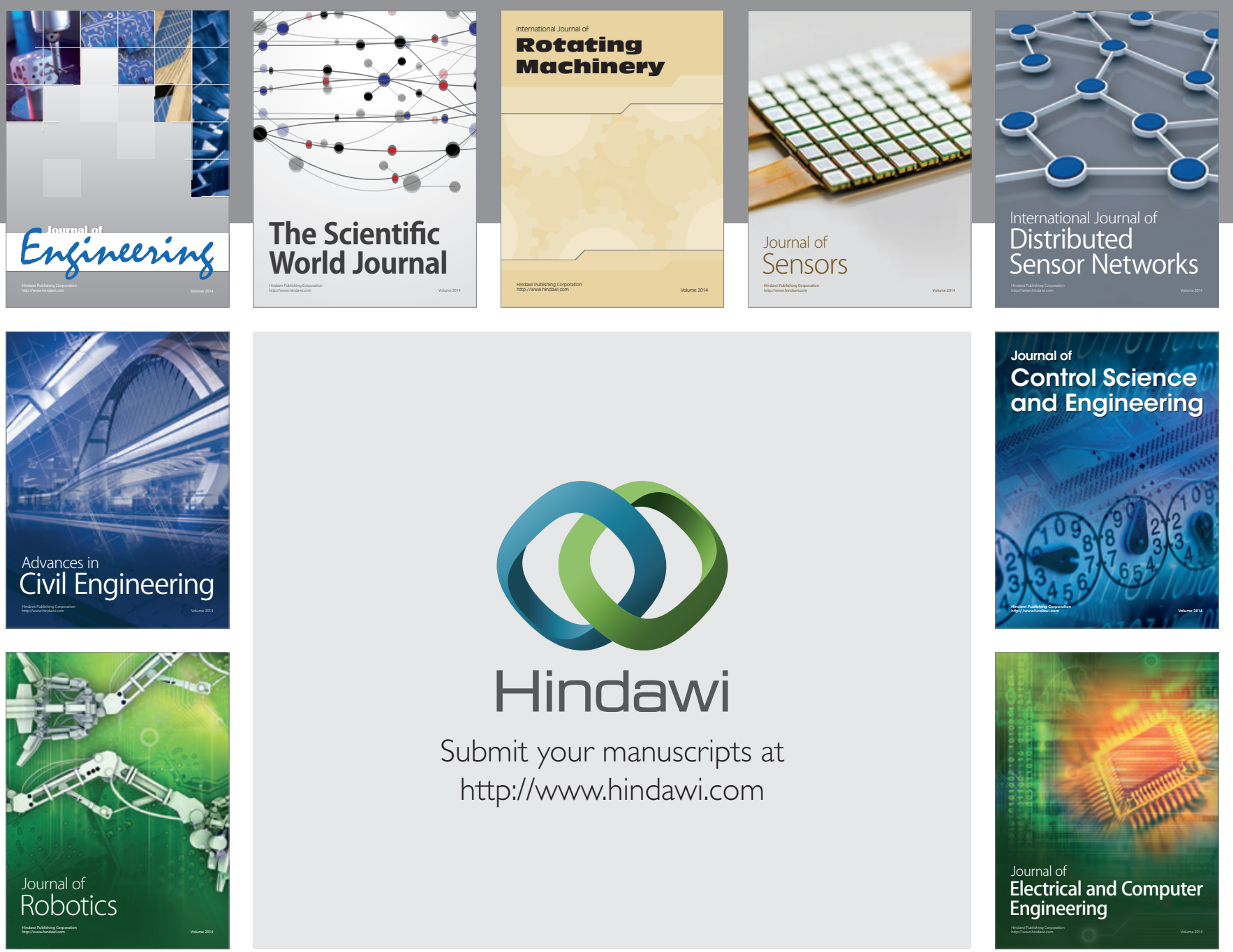

Submit your manuscripts at

http://www.hindawi.com
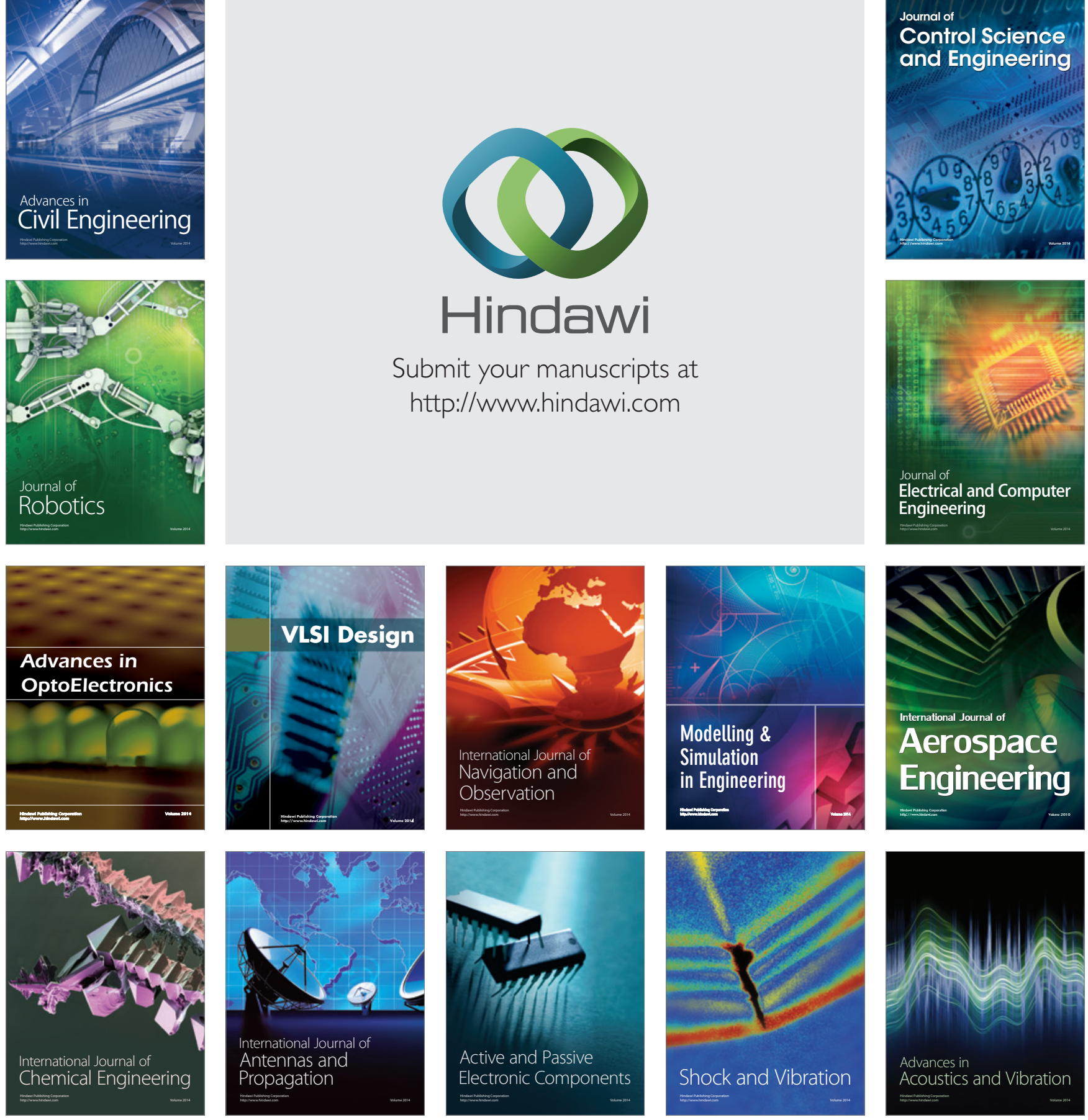